\title{
Study of cryogenic photomultiplier tubes for the future two-phase cryogenic avalanche detector
}

\author{
A. Bondar, ${ }^{a, b}$ A. Buzulutskov, ${ }^{a, b}$ A. Dolgov, ${ }^{b}$ E. Frolov, ${ }^{a, b}$ V. Nosov,,${ }^{a, b}$ L. Shekhtman, ${ }^{a, b, 1}$ A. \\ Sokolov ${ }^{a, b}$ \\ ${ }^{a}$ Budker Institute of Nuclear Physics, SB RAS, \\ 630090 Novosibirsk, Russia \\ ${ }^{b}$ Novosibirsk State University, \\ 630090 Novosibirsk, Russia
}

E-mail: L.I.Shekhtman@inp.nsk.su

\begin{abstract}
AвsTRACT: We report the results of a characterization study of several types of cryogenic photomultipliers manufactured by Hamamatsu Photonics and intended for operation in liquid Ar conditions, namely: compact 2-inch R6041-506MOD tubes, 3-inch R11065-10 and R11065-MOD tubes for operation in liquid Ar and 3-inch R11410-20 tubes originally designed for operation in liquid Xe. These types of PMT are proposed for installation into the future two-phase cryogenic avalanche detector that is developed in the Laboratory of Cosmology and Particle Physics of the Novosibirsk State University jointly with the Budker Institute of Nuclear Physics. Eight R11065 PMTs and seven R11410-20 tubes were tested and all demonstrated excellent performance in liquid $\mathrm{Ar}$ in terms of gain and relative single electron efficiency. All 3-inch PMTs showed a maximal gain in liquid Ar above $5 \times 10^{6}$ and relative single electron efficiency higher than $95 \%$. Compact R6041-506MOD tubes have dynode system different from that of the 3-inch photomultipliers and thus their single electron energy resolution and relative efficiency is much worse than that of 3-inch tubes. From 21 2-inch PMTs only 12 tubes were selected with acceptable, i.e. higher than 75\%, relative single electron efficiency and the maximal gain higher than $5 \times 10^{6}$. However, these PMTs are very attractive because they are the only compact type of tubes that can operate in liquid Ar.
\end{abstract}

KeYwords: Photon detectors for UV, visible and IR photons (vacuum) (photomultipliers, HPDs, others), Cryogenic detectors, Dark Matter detectors (WIMPs, axions, etc.)

${ }^{1}$ Corresponding author 


\section{Contents}

1 Introduction 1

2 Experimental set-up and conditions of the measurements 3

3 Results of the measurements 5

3.1 R11065-10, R11065-MOD 5

3.2 R6041-506MOD 7

3.3 R11410-20

3.4 Long-term performance of the cryogenic PMTs. 8

4 Conclusions $\quad 8$

\section{Introduction}

Two-phase Cryogenic Avalanche Detector (CRAD) is proposed and being developed in the Laboratory of Cosmology and Particle Physics of the Novosibirsk State University jointly with the Budker Institute of Nuclear Physics [1-5]. The main purpose of this detector is to search for weakly interacting massive particles (WIMP) [6] and coherent neutrino-nucleus scattering [7,8] by detecting signals from nuclear recoils [9]. The energy of recoil nuclei from WIMP is predicted not to exceed several tens of $\mathrm{keV}$ with the most interesting range below $8 \mathrm{keV}$ that corresponds to the WIMP mass below $10 \mathrm{GeV}$. For coherent neutrino-nucleus scattering the recoil energy is even lower, below 0.5 $\mathrm{keV}$. Thus the CRAD sensitivity that can allow detection of single primary electrons becomes a factor of crucial importance.

The design of proposed CRAD is shown schematically in Fig. 1. The detector consists of a cryogenic vessel with a vacuum thermal insulation that can hold up to 1501 of liquid $\operatorname{Ar}(200 \mathrm{~kg})$. The sensitive volume is limited by the height of the bottom PMT system as well as the thickness of side PMTs and is close to 501 (50 cm in height and $36 \mathrm{~cm}$ in diameter). Primary electrons, produced by recoil nuclei after WIMP or neutrino scattering, drift in the liquid towards the surface in the electric field of $2 \mathrm{kV} / \mathrm{cm}$. At the liquid surface electrons are emitted in the gas phase where they produce signal in two stages: first by electroluminescence in the high electric field above the liquid and second by avalanche multiplication in a double-THGEM or in a hybrid 2THGEM/GEM cascade.

Proportional scintillations in the VUV region from an electroluminescence (EL) gap (S2 signal) are detected by the ring of side PMT and by the bottom PMT system. To provide sensitivity in the VUV range all PMTs have a wavelength shifter (tetraphenyl-butadiene, TPB) deposited either directly on the PMT window or on a thin transparent plastic foil in front of them. The bottom PMTs are also intended for the detection of primary scintillations in the liquid (S1 signal). 


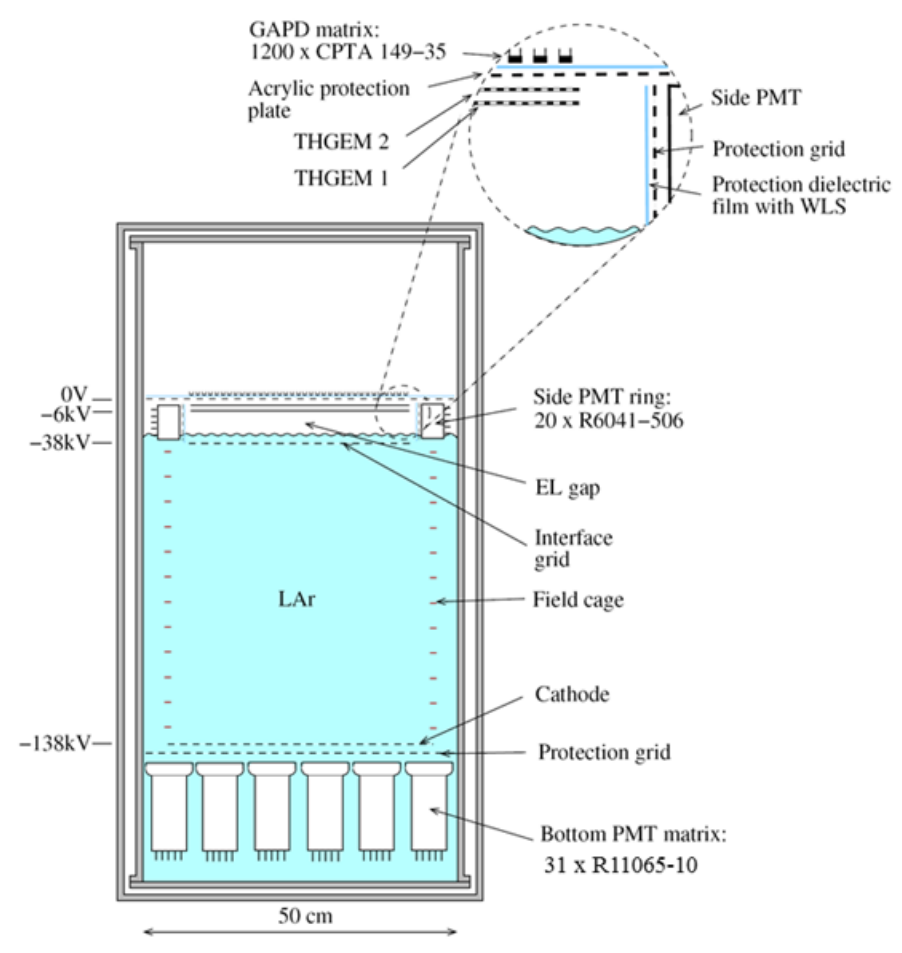

Figure 1. Design of two-phase CRAD in Ar with optical readout by a combined GEM/GAPD multiplier.

For the bottom PMT system an R11065 Hamamatsu 3-inch PMT was chosen based on the experience of WArP and DarkSide experiments [10, 11]. This PMT has a bialkali photocathode adapted for operation down to liquid Ar temperature ( $87 \mathrm{~K})$. The quantum efficiency $(\mathrm{QE})$ reported by the manufacturer is not less than $25 \%$ at $420 \mathrm{~nm}$. The authors of [10] report, however, the value of 33\% QE at $420 \mathrm{~nm}$. For the side PMT system the Hamamatsu R6041-506MOD device was chosen. This is a compact 2-inch PMT with a metal-channel dynode structure and a bialkali photocathode adapted for operation in liquid $\operatorname{Ar}(87 \mathrm{~K})$ with $25 \%$ QE at $420 \mathrm{~nm}$ [12]. This tube has $57 \mathrm{~mm}$ diameter and $43.5 \mathrm{~mm}$ height, which allows to keep the diameter of the sensitive volume not less than $36 \mathrm{~cm}$.

The TPB layer considerably reduces a photon collection efficiency, by a factor reaching 20 , due to internal reflection and conversion efficiency losses in the wavelength shifter (WLS) and because of the absence of optical contact between the WLS and the PMT window. Thus, it looks attractive to shift the VUV emission of Ar to a longer wavelength directly in the detection medium without the WLS. It is known that in liquid Ar such a VUV-to-UV conversion can be performed by doping with $\mathrm{Xe}$, at a content of 10-1000 ppm (see [13] and references therein). For this case we propose here to use a 3-inch R11410 PMT in liquid Ar conditions, originally designed for use in liquid Xe [14-17]. This PMT has an enhanced sensitivity to UV photons emitted by Xe, with a quantum efficiency close to $30 \%$ at $175 \mathrm{~nm}$ (Fig. 2) [18]). On the other hand, it has the same dynode system, the same body and the similar bialkali photocathode as those of R11065, which give hope for a 
successful operation at liquid Ar temperature.

Another motivation for testing an R11410 PMT in liquid Ar conditions comes from the idea of the "universal argon-xenon detector". In this idea, the detector designed for operation in liquid $\mathrm{Xe}$, in particular that of the RED experiment [17], can also be operated with liquid Ar using the same PMT system, thus diversifying the target material.

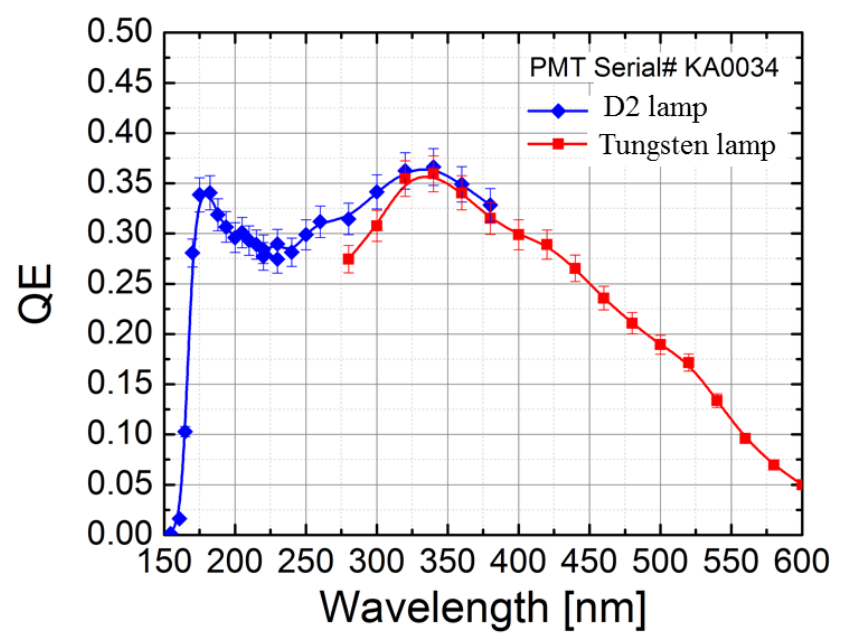

Figure 2. Quantum efficiency of R11410 PMT. Result of the measurement from [18].

\section{Experimental set-up and conditions of the measurements}

In our previous work excellent performance of the R11065-10 and R11065-MOD PTMs in liquid Ar was demonstrated [19] and these results are presented here for completeness. The total number of compact R6041-506MOD PMTs purchased from Hamamatsu Photonics is 21 pieces. The results of the performance of four PMTs were reported in [19]. In the present work the rest 17 PMTs are tested. Seven 3-inch R11410-20 PMTs are tested in the present work and for the first time their successful operation in liquid Ar is demonstrated. The image of R6041-506MOD and R11410-20 PMTs is shown in Fig. 3. The set-up for testing of PMT immersed in liquid Ar is presented in Fig. 4. For the tests of PMT we used a stainless steel vessel $30 \mathrm{~cm}$ in diameter and $40 \mathrm{~cm}$ high with a gas-tight top cover. The vessel can keep up to $3 \mathrm{~atm}$ of overpressure. The top cover contains three metal-glass feedthroughs that were used to provide high voltage to the PMT under test, signal from the pulse generator to the light emission diode (LED), signals from the temperature sensors to the Lake Shore 336 temperature controller and voltage to the heater from the same controller. Cryocooler Cryodyne 350CP by CTI-Cryogenics was used for cooling and liquefaction of Ar. The PMT under test was installed inside a thermal insulating cylindrical case $10 \mathrm{~cm}$ in diameter and 35 $\mathrm{cm}$ high. The case has a plastic PET (polyethylene terephthalate) bottle $8 \mathrm{~cm}$ in diameter and 30 $\mathrm{cm}$ high firmly attached inside. At the bottom the bottle has special support containing the LED for PMT cathode illumination. 


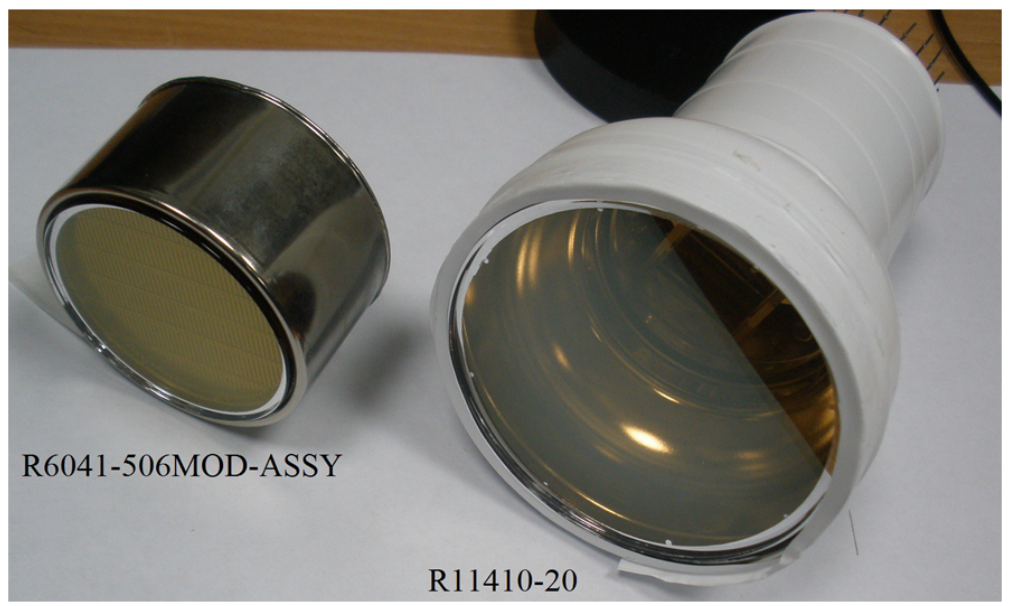

Figure 3. Photo of R6041-506MOD and R11410-20 photomultipliers.

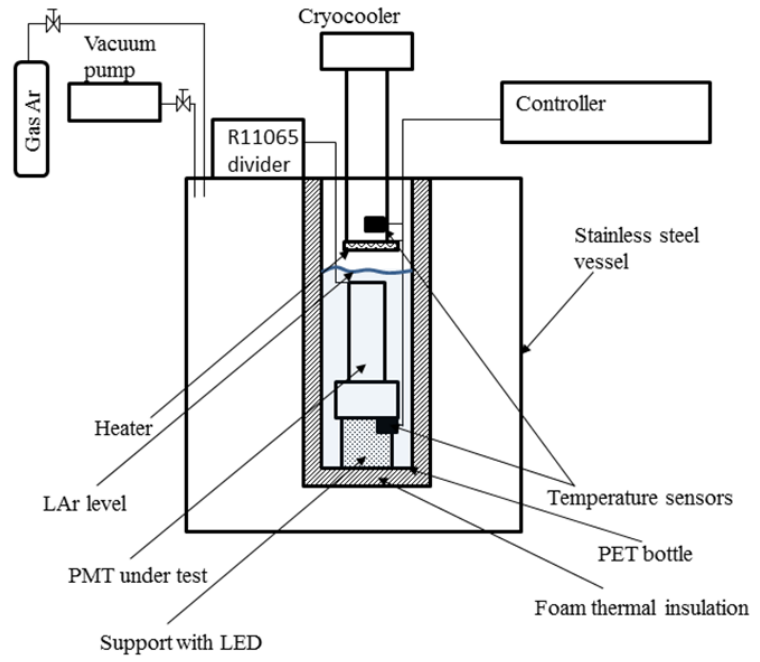

Figure 4. Schematic set-up for the PMT tests.

All three types of PMTs, R11065, R11410-20 and R6041-506MOD were powered by positive voltage from N1470 CAEN high voltage power supply. A signal from the PMT anode was amplified by a factor of 10 or 100 with a CAEN N979 fast linear amplifier module. Then amplified signal was connected to a Tektronix TDS5034B oscilloscope and to a discriminator CAEN N841 for counting rate measurements by a scaler CAEN N1145. The oscilloscope was used for the measurements of waveforms and pulse height spectra. Signals were obtained from spontaneous emission of single electrons from photocathode or by illumination with the LED. The LED was powered from a pulse generator, and its light intensity could be tuned by changing a pulse height and base line from the 
generator. The rate with LED did not exceed $5 \mathrm{kHz}$ and the frequency of the pulse generator was $50 \mathrm{kHz}$.

\section{Results of the measurements}

\subsection{R11065-10, R11065-MOD}

Single electron response (SER) of an R11065 tube is rather fast, FWHM of the signal is $7 \mathrm{~ns}$ and full width at 0.1 maximum is around $12 \mathrm{~ns}$. The gain can reach values close to $10^{7}$ and the single electron spectrum is very well separated from the noise (see Fig. 5). The gain of the PMT was calculated from the measurements of the pulse height spectra of SER. At first the waveform was measured for each PMT: by integrating it and expressing the total charge in electrons, the relationship between the full charge and the pulse height was calculated. Then the pulse height spectra were measured and the most probable pulse height was found for each spectrum by fitting with the Gaussian distribution. The pulse height distribution of SER in PMT in the absence of external noise sources is mainly determined by multiplication statistics of the first dynode that is well described by the Poisson distribution. The Poisson distribution with the average value from 7 to 9 (see [19]) is very close to that of the Gaussian. Moreover, the rest dynodes and electronic noise smear the final distribution following in average the Gaussian law. A gain as a function of anode

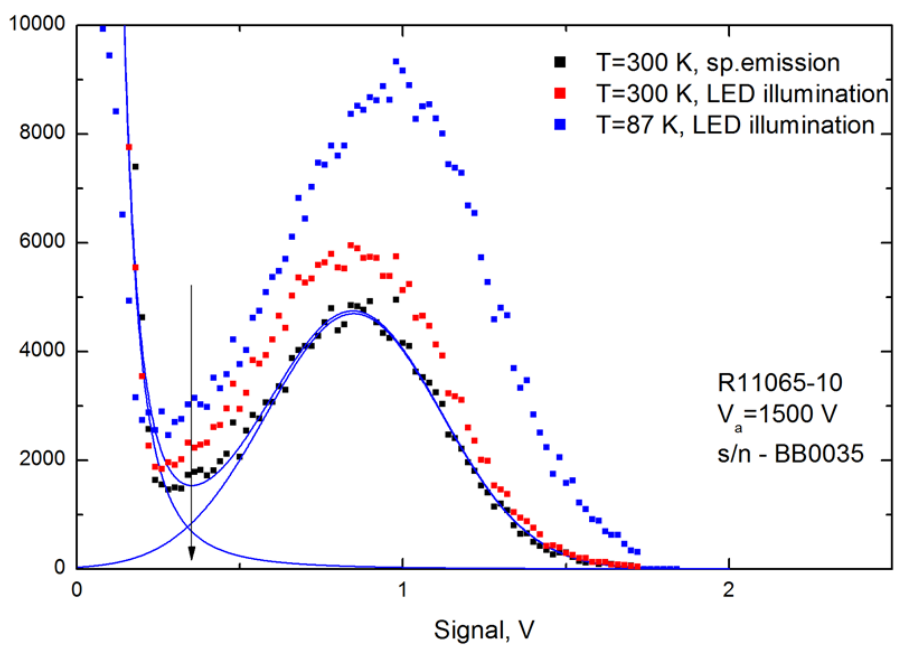

Figure 5. Pulse height spectra of SER obtained from spontaneous emission (black points) and with LED illumination at room temperature (red points) and at $87^{\circ} \mathrm{K}$ (blue points). Vertical arrow points to the threshold for calculation of the relative single electron efficiency. Anode voltage is $1500 \mathrm{~V}$ and the gain is $10^{7}$.

voltage is shown in Fig. 6 for three typical photomultipliers. A relative single electron efficiency (fraction of the SE response spectrum above threshold) reaches $95 \%$ at the gain below $5 \times 10^{6}$ for all photomultipliers (Fig. 7). 


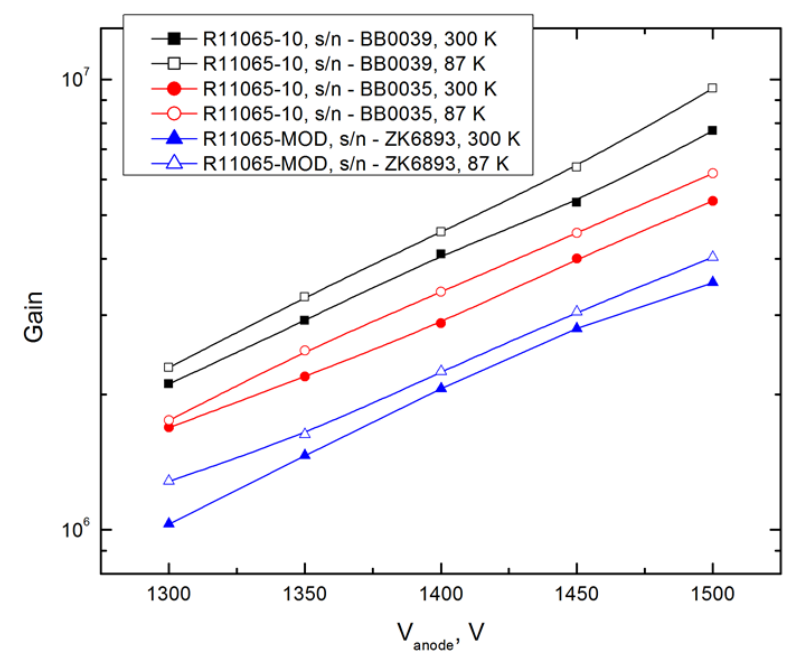

Figure 6. Gain as a function of anode voltage for two R11065-10 (one with the highest maximum gain and one with the lowest maximum gain) and one R11065-MOD PMT. The results for different PMTs are marked with different colors. The data corresponding to liquid Ar temperature are marked with open points.

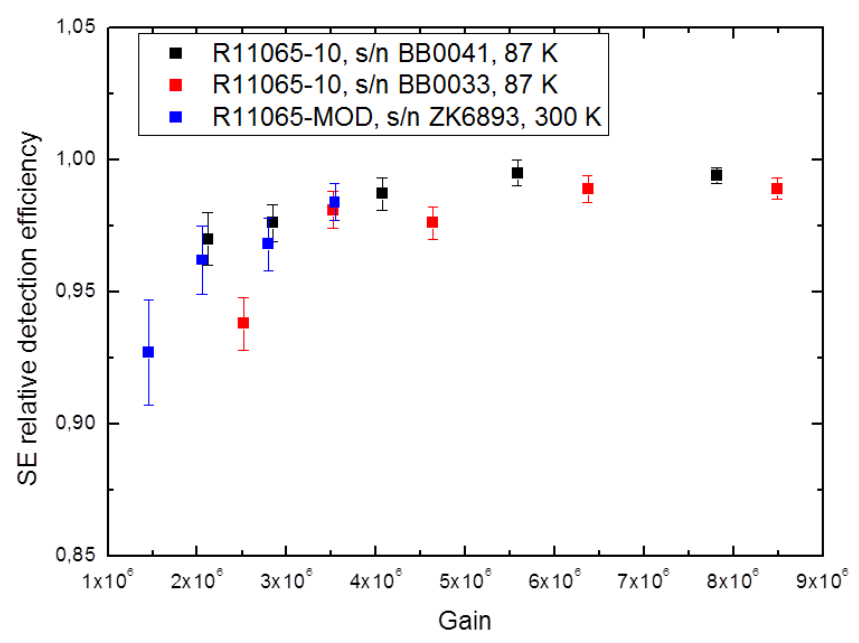

Figure 7. Relative detection efficiency of single electrons as a function of PMT gain. The data are shown for three PMTs with the lowest, medium and the highest maximum gain. 


\subsection{R6041-506MOD}

This PMT is about twice slower than R11065: FWHM of the waveform is 14 ns and the width at 0.1 of maximum is about $20 \mathrm{~ns}$. As R6041-506MOD is a slower PMT as compared to R11065, it has an approximately twice lower average SE signal at the same gain. A typical SE response spectrum is shown in Fig. 8. As in the case of R11065, linear dependence between the pulse height and total charge was used to calculate the gain. Since there was significant asymmetry of the SER spectrum, the Poisson distribution convolved with the Gaussian function was used for the signal spectrum approximation. The convolution allowed to account for the distribution smear due to the second- and higher-order dynodes and external noises. To account for the discriminator used as the trigger for the data collection, the signal model curve was multiplied by a steplike function with a narrow slope. Also the noise spectrum measured at zero voltage was added to the fitting curve of the SER spectrum. This noise was mainly caused by the electronics and was normally stable during the measurement runs and independent of anode voltage. The average pulse height was calculated from the fitted signal curve without considering discriminator cut off. The gain of

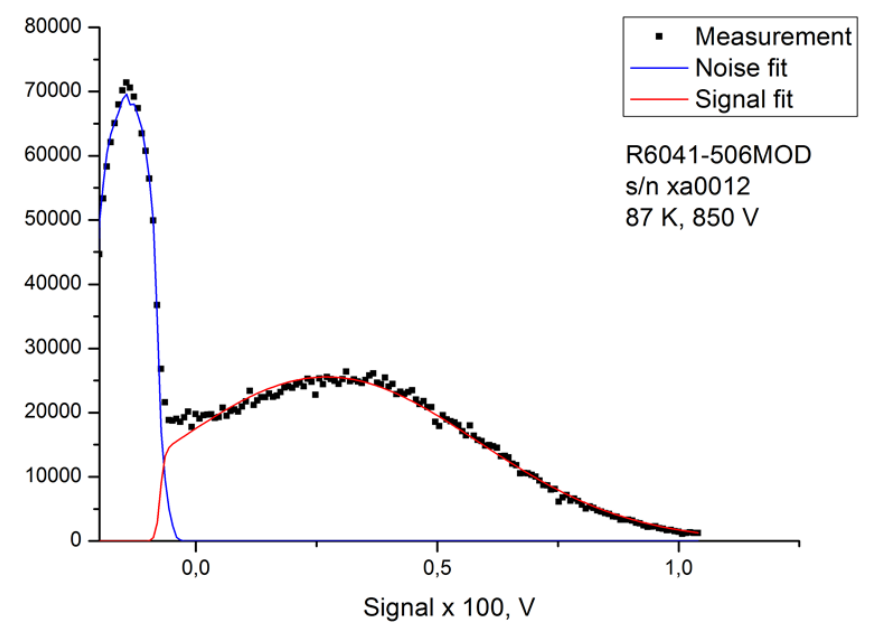

Figure 8. Typical pulse height spectrum of SE response for a R6041-506MOD tube from a group of the PMTs with higher gain.

R6041-506MOD can reach $10^{7}$, however, these tubes have rather large spread of maximum gain and relative SE efficiency. Only eight photomultipliers with relative SE efficiency higher than $75 \%$ could be selected (Fig. 9). In total only 12 PMTs out of 21 were selected for future operation in the two-phase CRAD.

\subsection{R11410-20}

Performance of these photomultipliers is very similar to that of R11065 tubes. As we did not know whether these tubes could operate without discharges in liquid Ar, the voltage was gradualy increased from $1000 \mathrm{~V}$ to $1500 \mathrm{~V}$ (recommended maximum voltage by the manufacturer). Four of the seven tested photomultipliers reached maximum voltage without discharges operating in liquid Ar. The other three PMTs exhibit discharges at the anode voltage between $1300 \mathrm{~V}$ and 

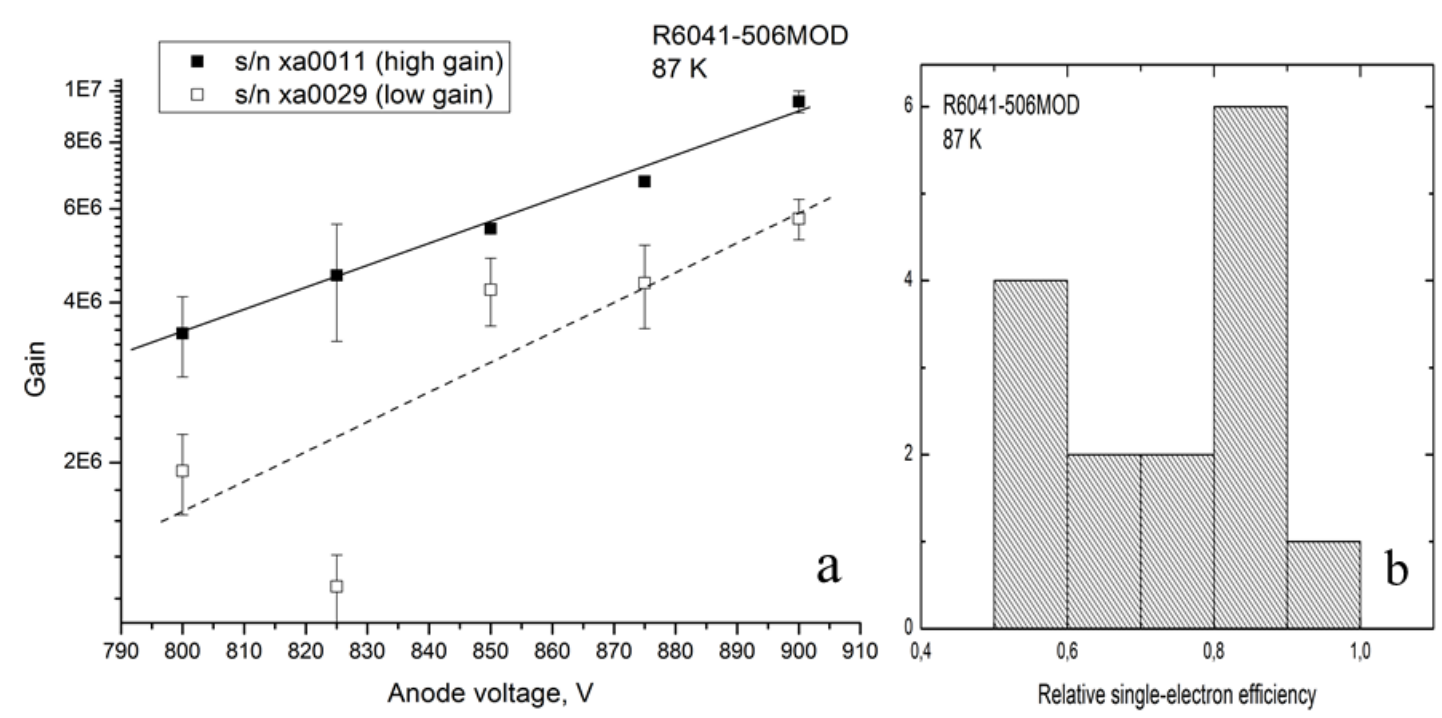

Figure 9. a) Gain as a function of anode voltage for two typical R6041-506MOD tubes, one from the group with a higher gain and another from the group with a lower gain. b) Distribution of PMT over the relative SE efficiency. Only eight tubes show relative efficiency exceeding $75 \%$.

$1375 \mathrm{~V}$ corresonding to the gain values around $3 \times 10^{6}$. After the discharges all parameters of the photomultipliers were recovered and anode voltage was kept below $1300 \mathrm{~V}$. The pulse shape and the pulse height spectra of R11410-20 tubes look very much like that of R11065. Spread of gains, energy resolutions and relative efficiencies is rather small, unlike in the case of R6041-506MOD tubes. Typical gain-voltage curves and relative efficiency vs gain dependencies are shown in Fig. 10 and Fig. 11. All seven tested photomultipliers demonstrated relative efficiency above $95 \%$ in liquid Ar.

\subsection{Long-term performance of the cryogenic PMTs.}

The behavior of one R6041-506MOD and one R11410-20 PMTs immersed in liquid argon for the period of several hours was studied. None of the gain, noise frequency or signal shape showed significant change for both tubes during the runs. Figure 12 shows gain vs time dependences for the R6041-506MOD tube and for R11410-20 photomultiplier. In both cases anode voltage was applied constantly during the whole run.

\section{Conclusions}

Operation of three types of the cryogenic PMT produced by Hamamatsu Photonics was tested in liquid Ar, namely 3-inch R11065-10 and R11065-MOD tubes, 2-inch compact R6041-506MOD tubes and 3-inch R11410-20 tubes sensitive to Xe UV emission. R11065 photomultipliers are designed for operation in liquid Ar. They demonstrate excellent performance, stable operation with 


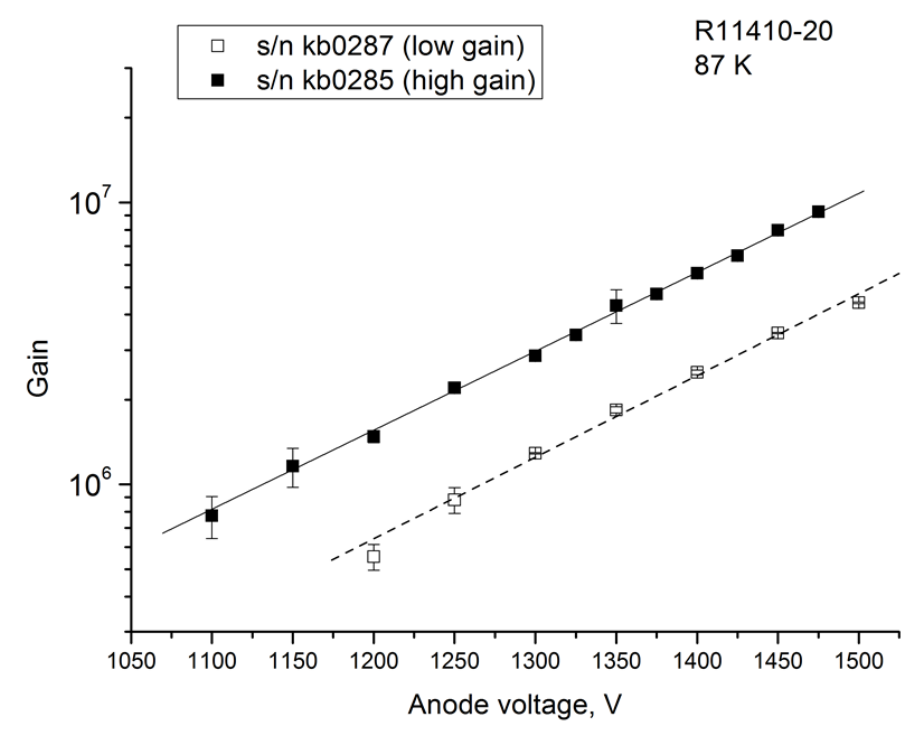

Figure 10. Gain as a function of anode voltage for R11410-20 PMT with the highest and the lowest gain in the tested group.

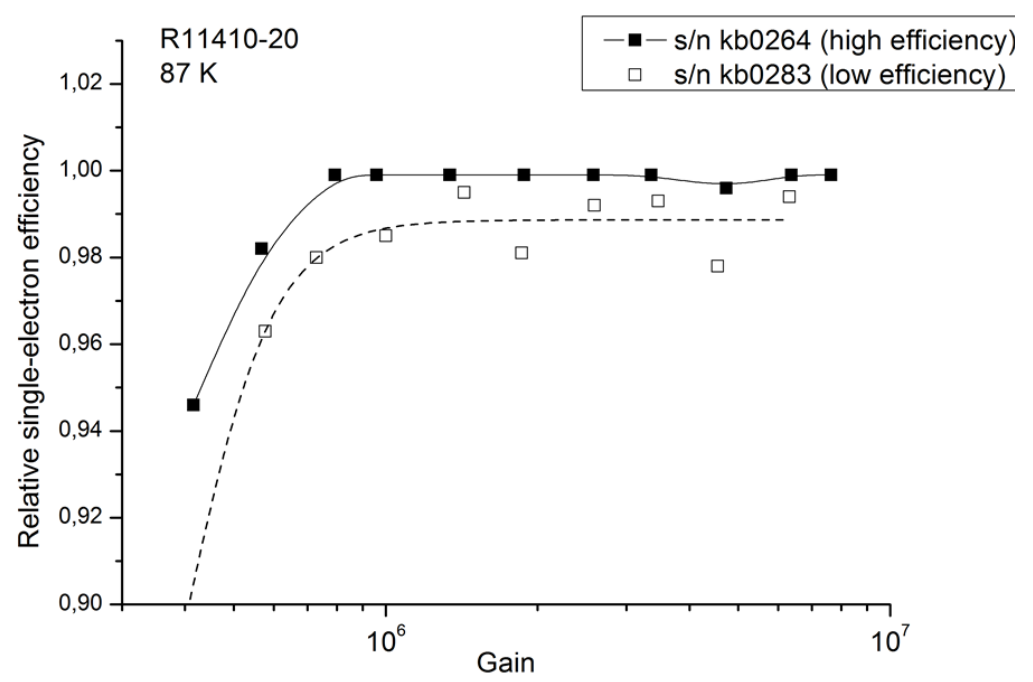

Figure 11. Relative SE efficiency as a function of gain for two R11410-20 tubes, one with the highest and one with the lowest efficiency from the tested group. 


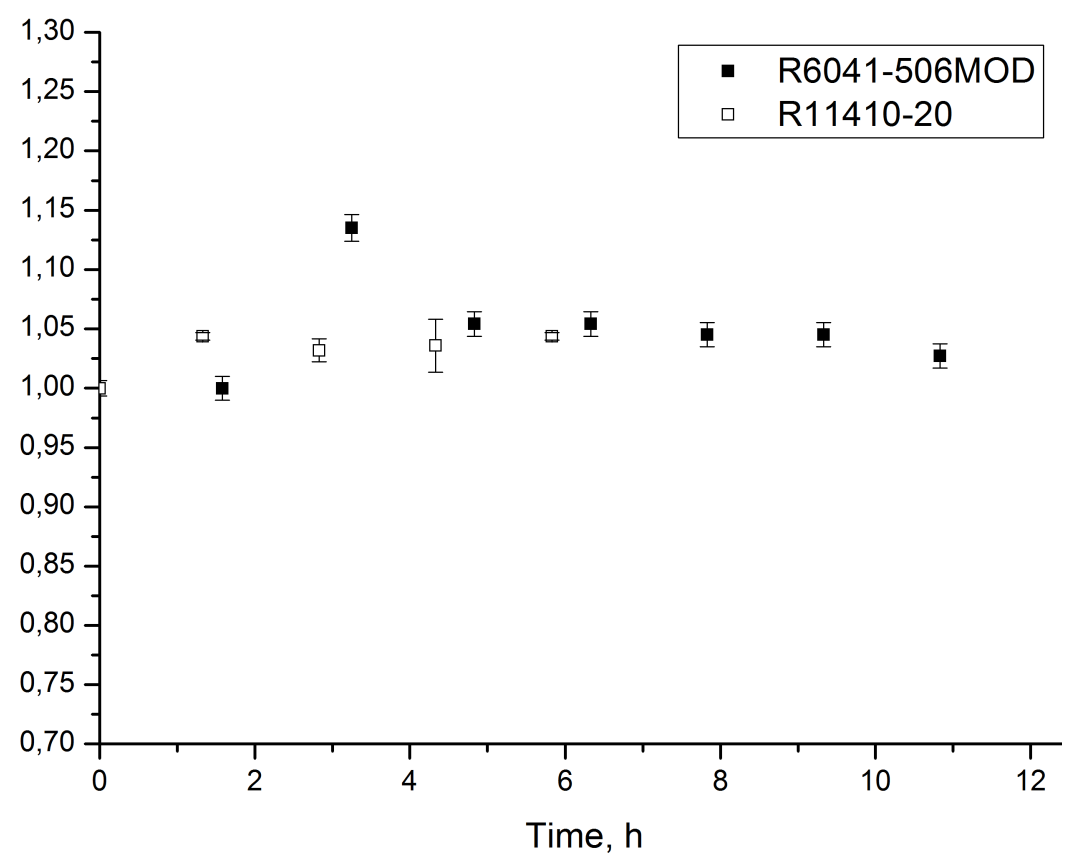

Figure 12. Relative gain as a function of time for R6041-506MOD and R11410-20 photomultipliers.

maximum gain reaching $10^{7}$ and relative efficiency for single electrons higher than 95\%. 2-inch R6041-506MOD tubes are very attractive due to their compact design, however their gain and relative efficiency exhibit high spread and only 12 photomultipliers out of 21 were selected with relative SER efficiency higher than 75\% for operation in future two-phase CRAD. R11410-20 tubes were designed for operation in liquid Xe and this work was the first demonstration of their operation in liquid Ar. These photomultipliers can safely operate at $87^{\circ} \mathrm{K}$ with a maximum gain up to $10^{7}$ and relative SER efficiency higher than $95 \%$.

\section{Acknowledgments}

This study was supported by Russian Science Foundation (project N 14-50-00080) and was done within the R\&D program of the DarkSide-20k experiment.

\section{References}

[1] A. Buzulutskov, Advances in Cryogenic Avalanche Detectors, J. of Instrum., 7 (2012) C02025.

[2] A.Bondar, et al., Study of infrared scintillations in gaseous and liquid argon. Part II: light yield and possible applications, J. of Instrum., 7 (2012) P06014.

[3] A. Bondar, et al., Proportional electroluminescence in two-phase argon and its relevance to rare-event experiments, Europhys. Lett., 112 (2015) 19001.

[4] A. Bondar, et al., Two-phase Cryogenic Avalanche Detector with electroluminescence gap operated in argon doped with nitrogen, Nucl. Instr. Meth. A, 845 (2017) 206. 
[5] A. Bondar, et al., MPPC versus MRS APD in two-phase Cryogenic Avalanche Detectors, J. of Instrum., 10 (2015) P04013.

[6] D. Akimov, Detectors for Dark Matter search (Review), Nucl. Instrum. Meth. A, 598 (2009) 275.

[7] C. Hagmann and A. Bernstein, Two-phase emission detector for measuring coherent neutrino-nucleus scattering, IEEE Trans. Nucl. Sci., 51 (2004) 2151.

[8] D. Akimov et al., Detection of Reactor Antineutrino Coherent Scattering off Nuclei with a Two-Phase Noble Gas Detector, J. of Instrum., 4 (2009) P06010.

[9] A. Bondar et al., Measurement of the ionization yield of nuclear recoils in liquid argon at 80 and 233 keV, Europhys. Lett., 108 (2014) 12001.

[10] R. Acciarri et al., Demonstration and comparison of photomultiplier tubes at liquid Argon temperature, J. of Instrum. 7 (2012) P01016.

[11] P. Agnes et al., First results from the DarkSide-50 dark matter experiment at Laboratori Nazionali del Gran Sasso, Phys. Lett. B, 743 (2015) 456.

[12] Hamamatsu Photonics, R6041-506MOD-ASSY Preliminary Data Sheet (2012)

[13] A.Buzulutskov, Photon emission and atomic collision processes in two-phase argon doped with xenon and nitrogen, Europhys. Lett., 117 (2017) 39002.

[14] K. Lung, et.al., Characterization of the Hamamatsu R11410-10 3-in. photomultiplier tube for liquid xenon dark matter direct detection experiments, Nucl. Instrum. and Methods A, 696 (2012) 32.

[15] D.S. Akerib, et.al., An ultra-low background PMT for liquid xenon detectors, Nucl.Instrum. and Methods A, 703 (2013) 1.

[16] L. Baudis, et.al., Performance of the Hamamatsu R11410 photomultiplier tube in cryogenic xenon environments, Journal of Instrum., 8 (2013) P04026.

[17] D.Yu. Akimov, et.al., Performance of Hamamatsu R11410-20 PMTs under intense illumination in a two-phase cryogenic emission detector, J. of Instrum., 11 (2016) P12005.

[18] A.Lyashenko, et.al., Measurement of the absolute Quantum Efficiency of Hamamatsu model R11410-10 photomultiplier tubes at low temperatures down to liquid xenon boiling point, J. of Instrum., 9 (2014) P11021.

[19] A.Bondar, et.al., Characterization of photo-multiplier tubes for the Cryogenic Avalanche Detector, J. of Instrum., 10 (2015) P10010. 